\title{
la flexión y los esfuerzos cortantes en el hormigón armado
}

ALFREDO PAEZ, Prof. Dr. ingeniero de caminos

$450-2$

\section{notación}

A = Sección de armaduras comprimidas.

$\mathrm{A}^{\prime}=$ Sección de armaduras en tracción.

$A_{t}=$ Armadura transversal (Sección de cercos verticales en una longitud de pieza igual al canto útil $h$ ).

$A_{v}=$ Armadura vertical (Cercos).

$A_{4,}=$ Barras levantadas a $45^{\circ}$ (Sección de barras en una longitud igual al canto útil, medida perpendicularmente a las barras).

$I=$ Momento de inercia.

$\mathbf{M}_{p}=$ Momento flector previsto.

$M=$ Momento límite.

$\mathbf{S}_{b}=$ Momento estático de la sección comprimida de hormigón.

$\mathrm{N}=$ Esfuerzo normal límite.

$\mathbf{N}^{\prime}{ }_{a}=$ Esfuerzo de tracción desarrollado por la armadura.

$\mathbf{N}_{b}=$ Esfuerzo de compresión ejercido sobre el hormigón.

$\mathrm{T}_{p}=$ Esfuerzo cortante previsto.

$\mathrm{T}=$ Esfuerzo cortante limite.

$b=$ Anchura de la sección.

$h_{1}=$ Canto total.

$h=$ Canto útil.

$h_{c}=$ Distancia entre baricentros de armaduras comprimidas y extendidas.

$z \quad=$ Brazo mecánico.

$\sigma_{b}=$ Resistencia del hormigón en compresión.

$\sigma_{b}^{\prime}=$ Resistencia del hormigón en tracción.

$\sigma^{\prime}{ }_{e}=$ Límite alástico del acero en tracción.

$\sigma_{e}=$ Límite elástico del acero en compresión.

$\psi=\frac{\mathrm{T} h}{\mathrm{M}}$.

$\tau=$ Tensión cortante.

\section{resumen}

En este artículo, el autor expone, de forma resumida, los principios y resultados que se obtienen al aplicar una teoria revolucionaria por el presentada en diversos Congresos y Organismos Internacionales, relativa al cáculo de las vigas de hormigón armado sometidas a fiexión simple. mento se apartan de la teoria clásica, y el hecho de que ya mento se apartan de la teoria clásica, y el hecho de que ya INFORMES DE LA CONSTRUCCION publique esta tesis que la experimentación parece confirmar plenamente.

Todos tenemos nuestros desasosiegos, nuestras dudas y vacilaciones. A veces, por esos pintorescos contrasentidos de la existencia, recibimos felicitaciones y enhorabuenas por algo que, en el fondo, nos ha dejado un poso de intranquilidad. 
No me refiero a nuestros cumpleaños, ni tampoco como alguien con malicia puede suponer, al nacimiento de un nuevo hijo. Es algo más trivial en el correr de los días.

Fué hace dos años, durante el Congreso Internacional del Hormigón Pretensado en Berlín (1958). Entre los trabajos presentados a la primera sesión figuraban dos míos. En uno de ellos, publicado en inglés, se exponían los resultados experimentales obtenidos en el ensayo de 16 vigas pretensadas, de $13 \mathrm{~m}$ de luz, rotas por flexión lenta en el Instituto Técnico de la Construcción y del Cemento. En el otro, redactado en castellano, se presentaba una nueva teoría sobre los esfuerzos cortantes en el hormigón.

Cuando una viga de hormigón armado se ensaya a rotura por flexión, la armadura situada en el borde extendido sufre positivos incrementos de carga a medida que aumentan las fuerzas exteriormente aplicadas. Llegado un cierto momento, la armadura alcanza el límite elástico. A partir de este instante, la pieza se deforma más y más, manteniéndose constante el esfuerzo desarrollado por la armadura.

El brazo mecánico aumenta, como único medio que le queda a la pieza para resistir los sucesivos incrementos de carga, hasta que, reducida la zona de compresiones del

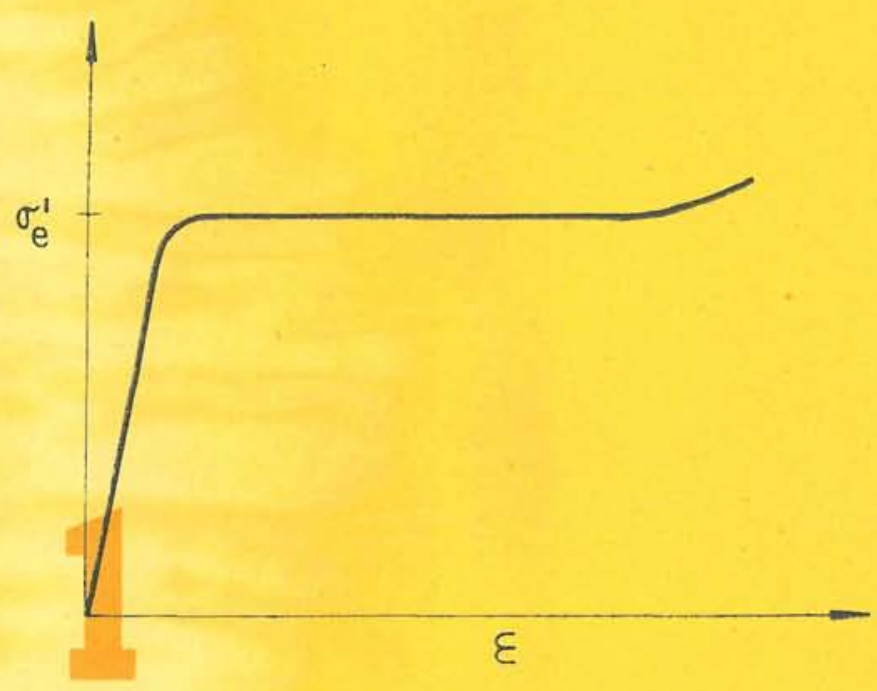
hormigón a un mínimo compatible con el esfuerzo de tracción desarrollado por la armadura, la pieza se rompe, incapaz de soportar nuevos aumentos de carga.

El cálculo estimatorio de la capacidad límite de resistencia de la pieza, se establece sobre la base de que la armadura, en esta fase final de rotura, desarrolla un esfuerzo de tracción igual al producto de su sección por el límite elástico. La verosimilitud de esta hipótesis, ampliamente refrendada por la experimentación, se justifica en la propiedad de los aceros ordinarios de presentar un tramo horizontal en el diagrama de tensión-deformación, a la altura del límite elástico (fig. 1). Alcanzado este límite, la barra se alarga con deformaciones impresionantes sin que la tensión sufra incrementos apreciables.

En las piezas de hormigón pretensado, el acero es de alta calidad. El diagrama de tensión-deformación de estos aceros, en lugar de presentar un definido escalón horizontal ofrece un tramo ligeramente incurvado de tensio-

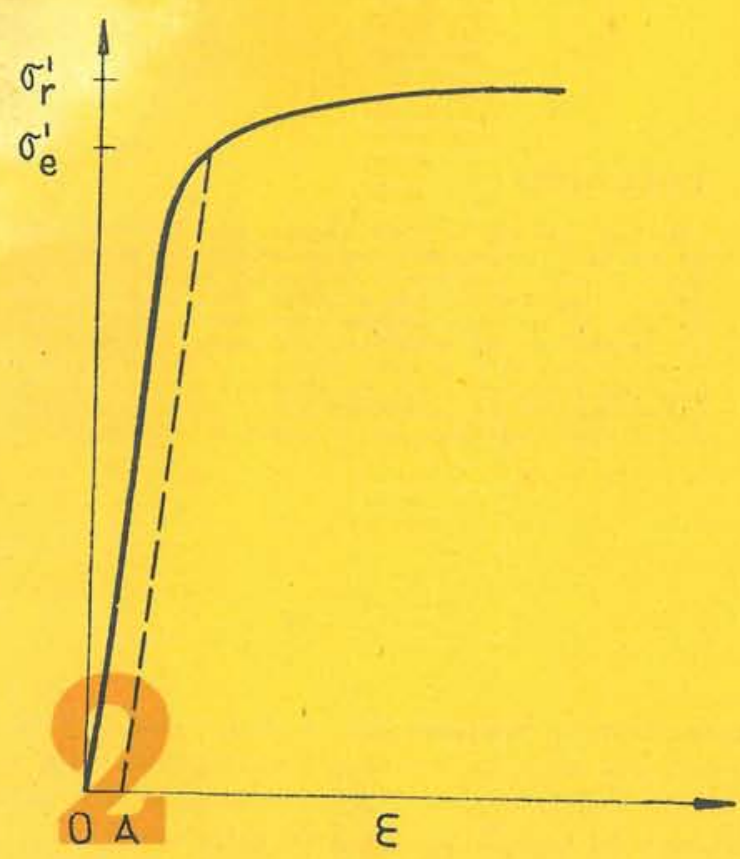
nes lentamente crecientes con la deformación. El propio concepto de límite elástico se desvanece, por impreciso, y para determinarlo se recurre al convenio de definirlo como aquella tensión $\sigma^{\prime}{ }_{e}$ que deja, al descargar la barra, una deformación remanente $\mathrm{OA}$ (fig. 2) igual al $0,2 \%$.

Así como en el caso del acero ordinario se comprende que sea el límite elástico la tensión alcanzada por el acero en la fase final de agotamiento resistente de la pieza, en el caso del hormigón pretensado, con sus aceros de alta resistencia, la duda prevalece por la característica forma del diagrama en el tramo final.

Los ensayos realizados sobre las 16 vigas pretensadas tenían como objetivo fundamental, precisar cuál es la tensión finalmente alcanzada por el acero de las armaduras pretesas, dato de gran interés para el cálculo de la capacidad límite de resistencia de las vigas pretensadas. 
Fuera por el interés de inmediata aplicación práctica de los resultados obtenidos, o fuera porque la publicación, en lengua inglesa, tuviera una mayor difusión por razones idiomáticas, el caso es que recibí muchas felicitaciones por el primer trabajo y muy pocas por el segundo, cuando, en realidad, éste tenía mucha más trascendencia que aquél.

No me extrañó.

Había leído en «Le petit Prince», de Antoine de Saint-Exupéry, la historia irreal, que entonces me parecía vivir, de un astrónomo turco que, en un Congreso Internacional, anunció la presencia de un asteroide hasta entonces desconocido. Nadie prestó atención a las afirmaciones de aquél que, vestido a la usanza oriental, más parecía un encantador de serpientes que un científico. Años después, cuando, renovada su indumentaria, se presentó en otro Congreso ataviado a la europea, obtuvo un éxito rotundo, nadie le regateó el aplauso y todos reconocieron la importancia de un descubrimiento que, antes, había pasado inadvertido.

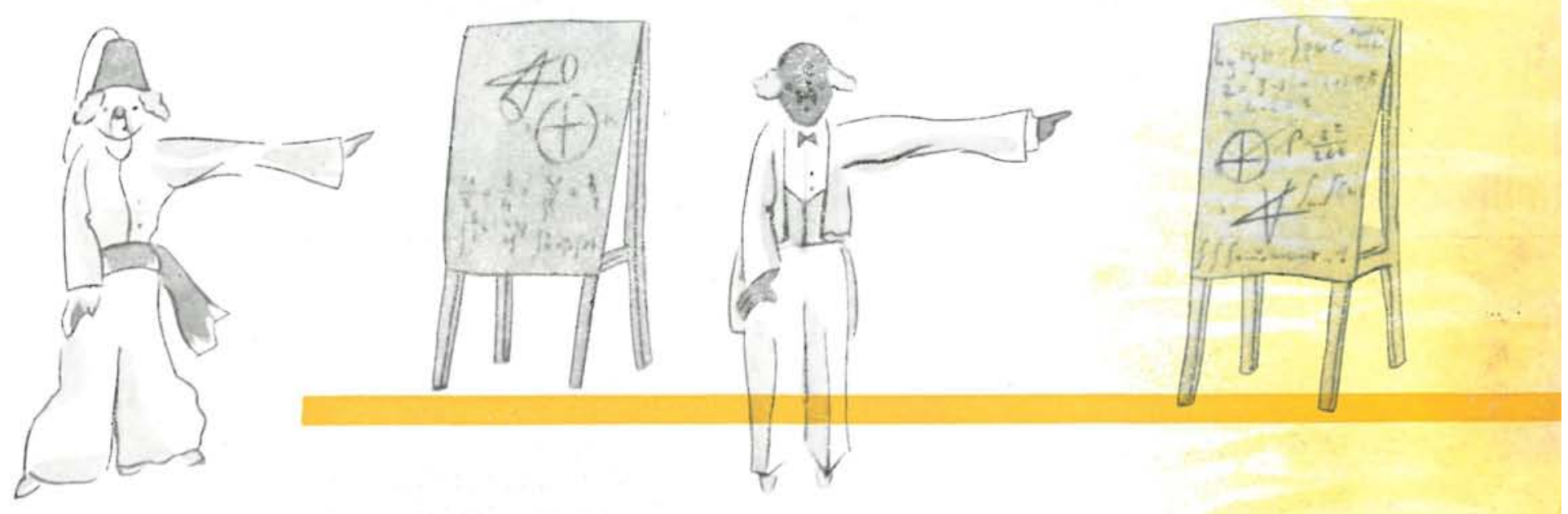

Las felicitaciones que en el Congreso de Berlín recibí no me halagaron. Intranquilo y pesaroso, recordaba que el segundo trabajo había nacido de las anomalías registradas en el primero. Sin aparente conexión, ambos estaban estrechamente ligados en un vínculo que parecía trivial. Los resultados obtenidos en el ensayo de las 16 vigas eran correctos, pero, y de ahí mis vacilaciones, no sé si había hecho recaer la atención lo suficiente sobre las zonas en donde se había producido la rotura.

Dato al parecer superfluo, pero llamado a tener su importancia.

El sistema de cargas empleado para romper las vigas, estaba formado por unos bidones colgados al extremo de unas palancas que ejercían la correspondiente presión sobre las piezas que se ensayaban. El número de cargas aplicadas era, en unos casos, cuatro, y en otros, seis, según la resistencia de las piezas. En todos ellos, las cargas se mantenían iguales, e incluso los incrementos de carga eran simultáneos gracias a una red de tuberías y grifos que permitían regular el caudal de agua que entraba, poco a poco, en cada uno de los bidones (figs. 3 y 4 ).

Las cargas aplicadas, simétricas en posición y magnitud, y en número par, dejaban un tramo central de $3,20 \mathrm{~m}$ en el cual el momento era prácticamente constante (esfuerzo cortante nulo), sin más variación que la debida al peso propio de la viga que se ensayaba; peso propio muy pequeño en comparación con las cargas que era necesario aplicar para alcanzar la rotura. En las figuras 5 y 6 se pueden observar los diagramas de momentos flectores y esfuerzos cortantes creados.

Siendo máximo el momento flector en el centro de la viga y constante las secciones de las armaduras longitudinal y transversal, los dispositivos para medir giros, deformaciones y flechas, se concentraron en los 3,20 m del tramo entre cargas centrales, para poder detectar las condiciones en que se producía la rotura (profundidad de fibra neutra, deformaciones extremas del hormigón, etc.). 


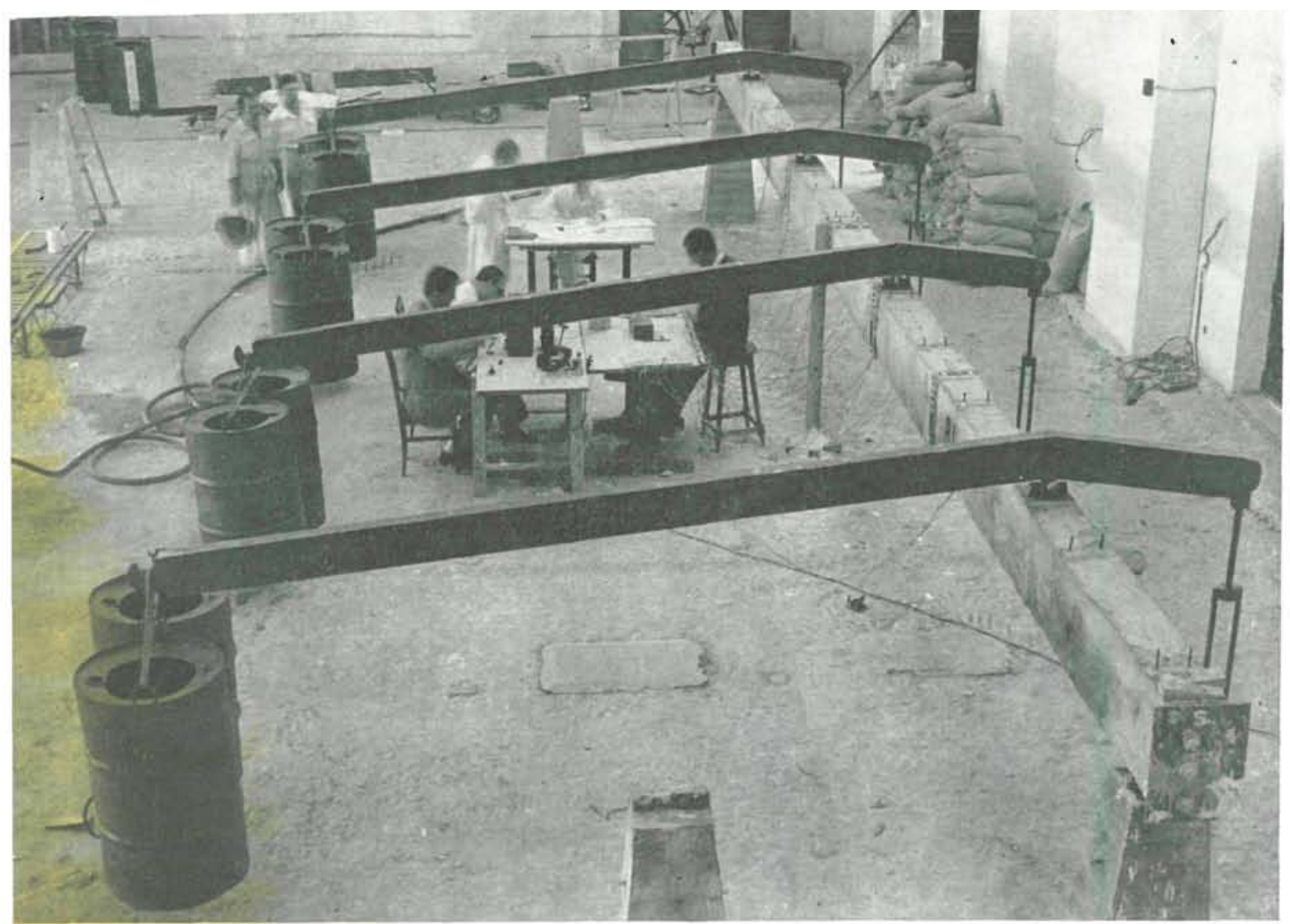

Con espíritu burlón, la primera viga, traviesa y femenina, se rompió por fuera del tramo central, en una sección inmediatamente situada por fuera del trozo limitado por las dos cargas centrales, como queriendo mantener el secreto de su desfallecimiento final. El momento flector de rotura, la flecha en el centro del tramo y los giros de los extremos quedaron, todos ellos, registrados, no pudiéndose determinar la deformación límite del hormigón en la sección de rotura, así como la probable posición de la fibra neutra.

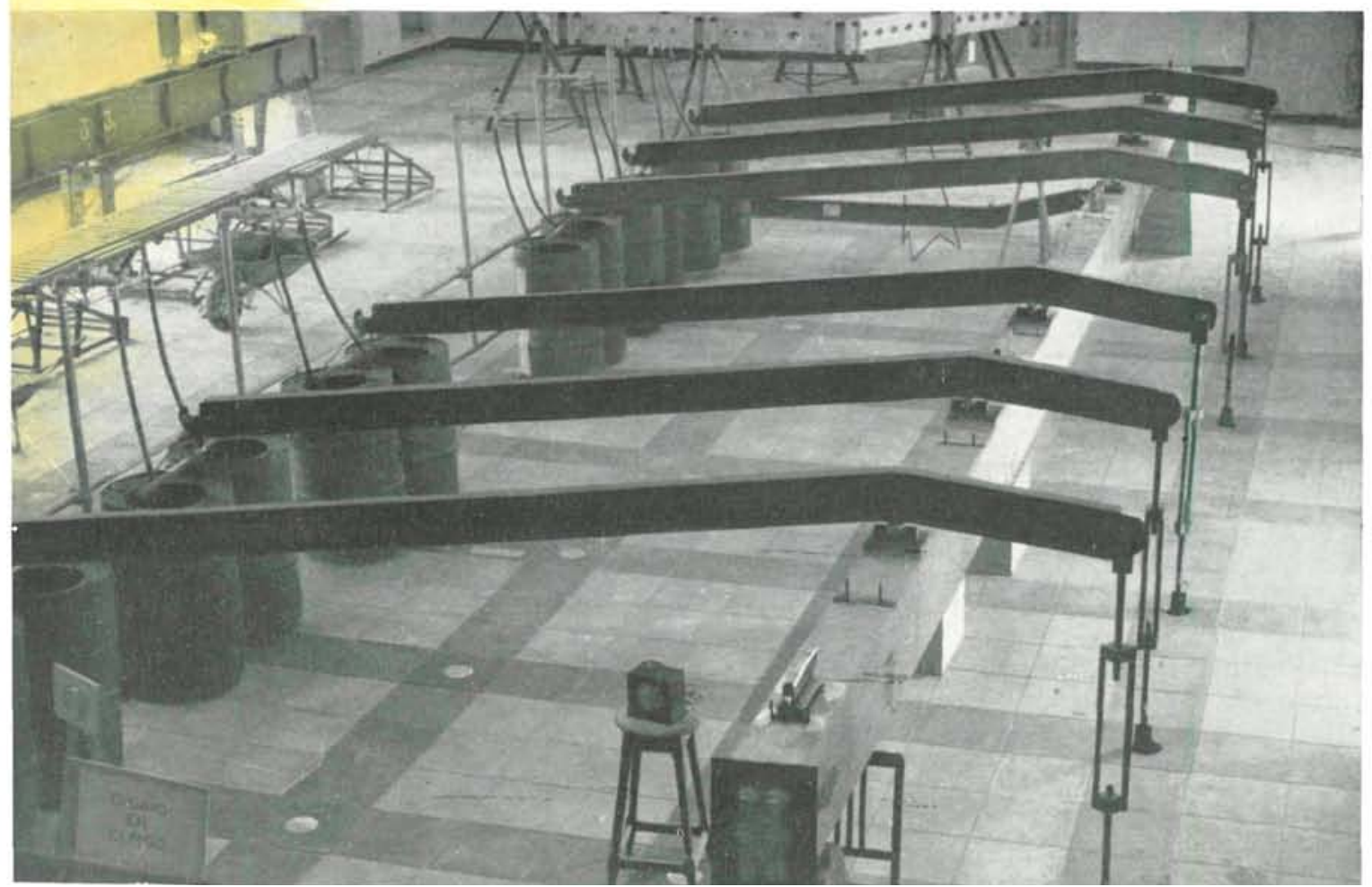



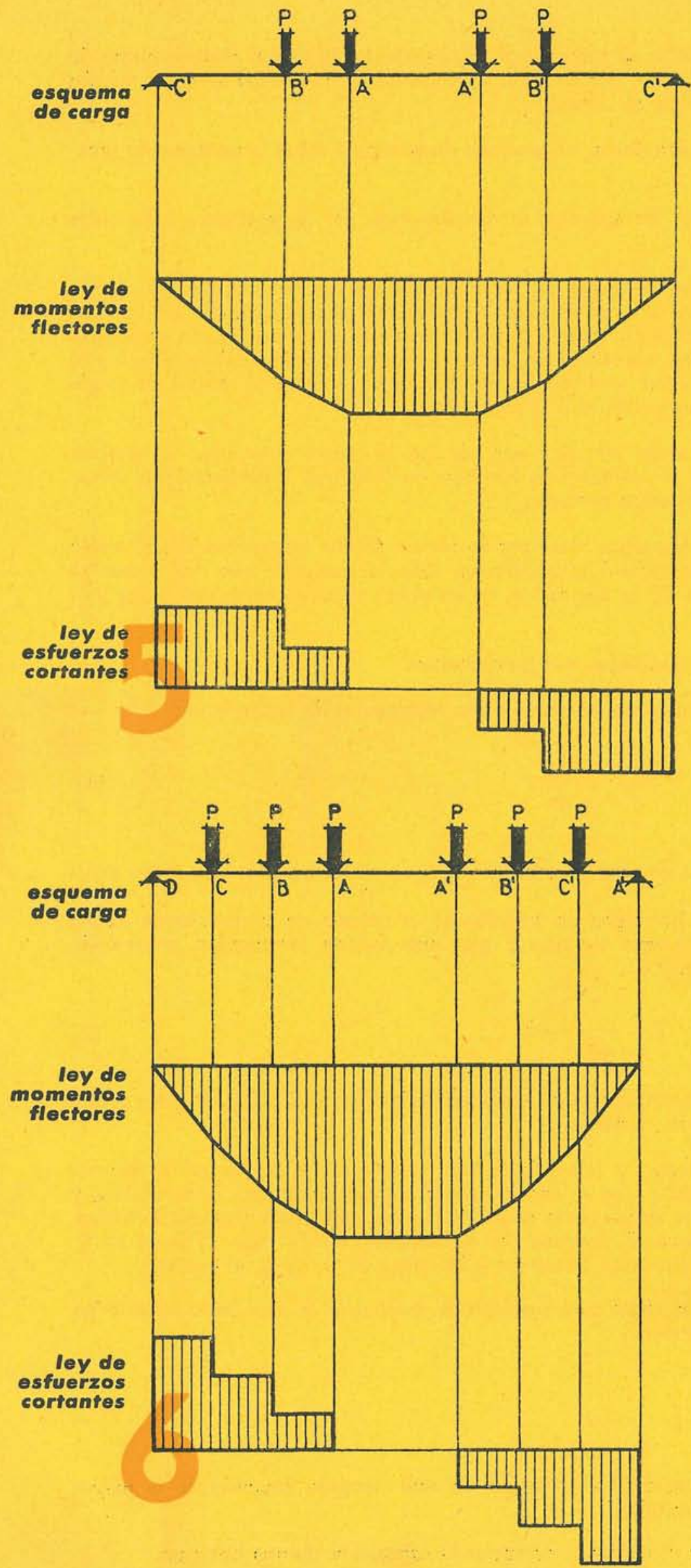

Sólo debe proponerse un cambio cuando esté plenamente justificada la incapacidad de los métodos de cálculo usuales en la representación de un cierto fenómeno.

Una teoría puede representar con acierto una fase del proceso resistente, por ejemplo los estados de prefisuración, sin que, por su origen, sea aplicable a otros regímenes más avanzados, como puede serlo el de prerrotura. Las hipótesis que en un caso concuerdan con la realidad, pueden ser dudosas en otros.

La teoría clásica establece, como cláusula principal, la homogeneidad y continuidad de un material ideal, cuyas deformaciones son proporcionales a las tensiones. Bajo estados moderados de carga, esta hipótesis es muy aproximada; pero cuando la pieza armada evoluciona en las proximidades de la rotura, el acero acepta fuertes deformaciones plásticas sin sensibles incrementos de tensión, al tiempo que las fisuras que aparecen en el hormigón rompen todo vestigio de continuidad y se producen profundas redistribuciones de esfuerzos, reajustes que ocasionan deformaciones que ni son elásticas, ni mantienen proporcionalidad alguna con las tensiones.

Sin ánimo destructivo, y con el único fin de establecer los límites de aplicación de cada teoría se resumen, en este apartado, las hipótesis que constituyen la base de la fórmula [1]:

a) El brazo mecánico:

$$
z=\frac{\mathrm{I}}{\mathrm{S}_{\mathrm{b}}},
$$

en donde I es el momento de inercia de la sección y $\mathrm{S}_{b}$ el momento estático de la sección comprimida de hormigón, respecto a la fibra neutra, es constante en cada sección $\mathrm{y}$, por consiguiente, es independiente de la magnitud de las solicitaciones aplicadas.

b) La pieza se rompe cuando la tensión principal de tracción $\sigma^{\prime}$ II alcanza la resistencia en tracción simple del hormigón, o cuando la tensión en el borde comprimido alcanza el valor de la resistencia en compresión $\sigma_{b}$.

c) Las tensiones de compresión en el hormigón en las diferentes alturas, son proporcionales a la distancia a la fibra neutra. La posición de esta fibra es, por otra parte, invariable. 
Cuando la segunda de las vigas ensayadas se rompió por una sección análoga, un silencio profundo siguió al crujido final que acompañó al suave descenso de la pieza. Como en el caso anterior, ningún defecto local pudo observarse en la sección de rotura.

El sistema de aplicación de las cargas exteriores, no arrojaba duda alguna sobre la permanente simetría de las cargas.

En el tercer ensayo, y en los siguientes, los aparatos se distribuyeron por las secciones adyacentes a la central.

No había duda, las vigas preferían romperse fuera del tramo central, en las secciones en que siendo el momento muy grande, aunque no el máximo, el esfuerzo cortante era considerable por ser igual al valor de la carga, $\mathrm{P}$, aplicada en cada palanca, más el pequeño aumento del peso propio correspondiente. Las secciones centrales de máximos momentos con esfuerzos cortantes despreciables (figs. 5 y 6 ) no parecían agotar tanto la pieza como estas secciones de los tramos adyacentes al central en donde la acción conjunta de ambas solicitaciones promovían la rotura final.

En esta primera serie de vigas de $13 \mathrm{~m}$ de luz, sólo una, de las 16 piezas ensayadas, se rompió, con estrépito, por la sección central. Las 15 restantes lo hicieron en secciones inmediatamente situadas fuera del tramo limitado por las dos cargas centrales.

En una segunda serie de vigas más pequeñas rotas por la acción de dos cargas situadas al tercio de la luz, con armadura transversal y longitudinal invariable en toda la longitud, sólo dos piezas se rompieron entre las dos cargas. Las otras 68 se rompieron en secciones situadas entre una carga y el apoyo.

Con arreglo a la teoría actual, estos resultados son inexplicables.

Según el cálculo, la tensión cortante máxima en una sección rectangular de ancho $b$, es:

$$
\tau=\frac{\mathrm{T}}{z \cdot b}
$$

siendo $z$ el brazo mecánico. Como norma general se admite que $z=\frac{7}{8} h$ (siendo $h$ el canto útil); y si se supone que la resistencia $\sigma_{b}^{\prime}$ del hormigón en tracción es el décimo de su resistencia $\sigma_{b}$ en compresión, se deduce que el máximo esfuerzo cortante $\mathrm{T}$ que una sección rectangular, no armada con cercos ni barras inclinadas, puede resistir es:

$$
\mathrm{T}=\frac{7}{80} \sigma_{b} b h .
$$

Los resultados experimentales han demostrado la incapacidad de las fórmulas [1] y [2] para representar la rotura de las piezas por esfuerzo cortante.

Las series más extensas están recopiladas, o han sido desarrolladas por la Universidad de Illinois. El $75 \%$ de las vigas de sección rectangular que se ensayaron sin colocar armaduras transversales, y con una armadura longitudinal superior a la necesaria para absorber los momentos flectores de rotura, se rompieron bajo cargas inferiores al esfuerzo T, previsto por la fórmula [2]. De este $75 \%$, el $42 \%$, prácticamente la mitad, se rompió bajo esfuerzos inferiores a la mitad de la carga $\mathrm{T}$ prevista.

Las fórmulas [1] y [2] no son las apropiadas para predecir la resistencia de una pieza cuando los esfuerzos cortantes se combinan con la flexión.

\section{Las hipótesis de la teoría clásica}

Toda fórmula tiene un campo de aplicación. La extensión de este dominio depende de la mayor o menor universalidad de las hipótesis admitidas.

No se establece una nueva teoría por el capricho de variar la exposición de una doctrina. 

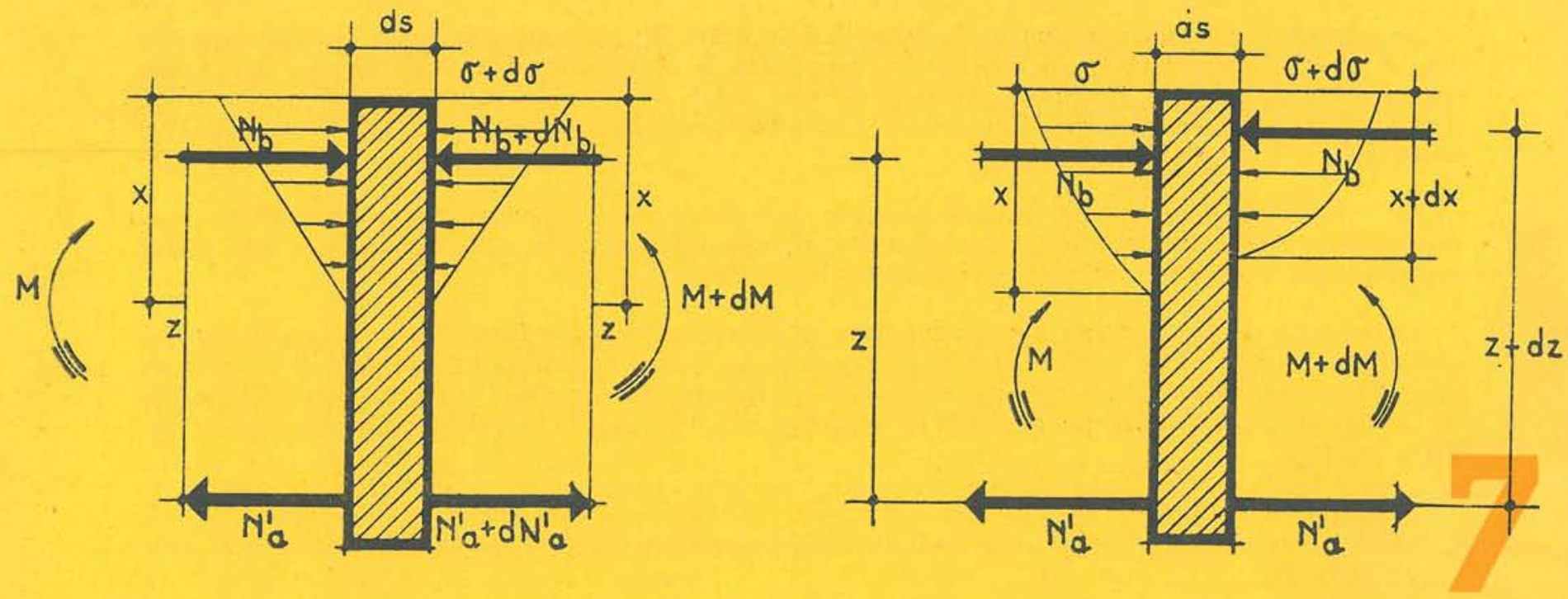

feoría clásica

Hipótesis a)

1. EI brazo mecánico es independiente de $\mathbf{M}$.

La profundidad $\alpha x \neq$ de la fibra neutra es invariable.

3. El esfuerzo $\mathbf{N}_{a}^{\prime}=\mathbf{N}_{b}$ depende de $\mathbf{M}$.

\section{nueva peoría}

Hipótesis a)

1. El brazo mecánico ez» varia con el momento $M$.

La posición de la fibra neutra ex» depende de la magnitud del

3. En las secciones próximas a la de rotura, el esfuerzo $\mathbf{N}_{a}=\mathbf{N}_{b}$ es constante.

\section{Las hipófesis de una nueva feoría}

Con el fin de poder explicar las aparentes anomalías de los resultados experimentales, y hallar unas fórmulas capaces de predecir con mayor precisión la resistencia real de las vigas de hormigón armado, se propone una nueva teoría cuyos principios se basan en las siguientes hipótesis:

a) En las proximidades de la rotura, cuando el hormigón está fisurado, la armadura longitudinal de tracción alcanza su límite elástico entre los bordes de la fisura abierta en la pieza. Unos deslizamientos aparecen entre el hormigón y el acero en las inmediaciones de la fisura. El esfuerzo de tracción $\mathrm{N}_{a}^{\prime}$ desarrollado por la armadura, igual a $\mathrm{A}^{\prime} \sigma^{\prime}{ }_{e}$ (sección por límite elástico), se mantiene constante en estas inmediaciones. En la sección de rotura, el esfuerzo de compresión $\mathrm{N}_{b}$ ejercido sobre el hormigón es, por consiguiente, constante entre dos secciones infinitamente próximas. Siendo constante este esfuerzo, el incremento $d \mathrm{M}$ de momento flector se consigue mediante un incremento $d z$ en el brazo mecánico, que, por consiguiente, varía con la magnitud de las cargas aplicadas, en la fase final de prerrotura (fig. 7).

b) Si una probeta de hormigón se somete a un ensayo de compresión, se rompe bajo la tensión $\sigma_{b}$. $\mathrm{Si}$ el ensayo es de tracción, la rotura se produce para una carga unitaria $\sigma_{b}^{\prime}$. Cuando se ensaya una probeta a compresión en una dirección y a tracción en otra dirección perpendicular a la anterior, la probeta se rompe por la acción simultánea de los dos esfuerzos cuando la tensión de compresión es inferior a $\sigma_{b}$ y la de tracción también inferior, en valor absoluto, a $\sigma_{b}^{\prime}$ (fig. 8).

La disminución de resistencia depende de la relación entre los esfuerzos de compresión y tracción ortogonales aplicados. La rotura no puede decirse que ha sido producida por la tracción o por la compresión. Fué.

c) Cuando se calcula o dimensiona una sección para que resista un momento flector, un esfuerzo normal o un esfuerzo cortante, la solución depende del diagrama o reparto de tensiones que se acepte. La hipótesis consistente en admitir una distribución rectangular o constante de tensiones en toda la zona comprimida, conduce a una máxima estimación del momento flector que la sección puede resistir, debido a la mayor compacidad del bloque de compresiones y, por consiguiente, a una máxima valoración del brazo mecánico. Por el contrario, una distribución triangular supone un reparto más suave de tensiones cortantes, con lo que se aumenta la capacidad teórica de la pieza frente a estos esfuerzos. 
Se admite, como hipótesis, que el hormigón elige, entre las diversas soluciones posibles, aquella que le permite aceptar mejor la solicitación compuesta. Si el momento flector es grande, comparado con el esfuerzo cortante (secciones centrales de una viga), la solución más favorable es la de un reparto rectangular de tensiones. Si el esfuerzo cortante predomina, adopta una distribución lineal o triangular.

En consecuencia, se admiten, simultáneamente, las distribuciones rectangular y triangular, como diagramas extremos, adoptándose, entre ellas dos, aquella que, en cada caso, conduzca a una mayor capacidad de resistencia, aceptándose siempre la hipótesis a) (fig. 7).

La primera de las hipótesis enunciadas tiene una justificación experimental directa. Las fisuras progresan y ascienden tanto más, cuanto más aumentan las cargas aplicadas. Las lecturas de los «straingages» y de los elongámetros mecánicos colocados a diferentes alturas de la pieza, acusan la elevación de la fibra neutra a medida que aumenta el momento, una vez pasada la primera fase que puede llamarse elástica.

La segunda hipótesis no es más que el enunciado, de un modo más o menos preciso, de la llamada «curva de resistencia intrínseca». Los resultados experimentales disponibles, permiten definir la relación enunciada.

La tercera hipótesis no es más que una generalización de la llamada «hipótesis a)». Deduciéndose los resultados que se obtienen según una y otra distribución, se observa que la experimentación coincide con el mayor de los resultados obtenidos.

\section{Piezas de sección rectangular sin armaduras transversales}

Por ser motivo de otra publicación más extensa, tanto la justificación de las hipótesis enunciadas como la deducción matemática de las fórmulas obtenidas, sólo se exponen, ahora, los criterios básicos que se han admitido en el planteamiento de la tesis que se desarrolla.

En el caso de la flexión pura, cuando el esfuerzo cortante es nulo, la rotura de la pieza armada se produce por aplastamiento del hormigón, cuando la máxima tensión longitudinal $\sigma_{M}$ alcanza el valor de la resistencia $\sigma_{b}$ del hormigón.

Cuando simultáneamente a la compresión ejercida por la flexión actúan unas tensiones cortantes $\tau$, derivadas del esfuerzo transversal $\mathrm{T}$, el hormigón debe resistir unas tensiones principales $\sigma_{I}$ y $\sigma_{\text {II }}^{\prime}$, la primera de compresión (sin acento) y la segunda de tracción (con acento), función de las tensiones $\sigma_{\mathrm{M}}$ y $\tau$.

Bajo un estado tan desfavorable de tensión doble, el hormigón se rompe antes de que $\sigma_{M}$ alcance la tensión $\sigma_{b}$ representativa de la resistencia en compresión simple $\left(\sigma_{I I}^{\prime}=0\right)$ del hormigón.

G. M. Smith, basándose en los resultados deducidos de un corto número de ensayos de compresión y flexión transversal, esiablece la relación empírica:

$$
\sigma^{\prime}{ }_{\mathrm{II}}=\mathrm{K} \sqrt{\sigma_{b}{ }_{b}-\sigma^{2}}
$$

Las series experimentales, posteriormente desarrolladas por McHenry y J. Karni, y especialmente, las más modernas y extensas de Bresler y Pister, así como las de Veriguin, han permitido confirmar la ley de Smith, sustituyendo el valor de $\mathrm{K}=0,1$, por él propuesto, por el de $\mathrm{K}=0,09$.

La ecuación [4] expresa así la condición de que para una tensión de compresión $\sigma_{I}$ menor que $\sigma_{b}$, existe una tensión principal de tracción $\sigma_{I l}^{\prime}$ también menor que la resistencia $\sigma_{b}^{\prime}$, supuesta igual a $0,09 \sigma_{b}$, que produce, junto con la $\sigma_{\mathrm{I}}$, la rotura del material.

Cuanto mayor es el esfuerzo cortante $\mathrm{T}$, mayor es la tensión $\tau$, mayor también es $\sigma_{I I}^{\prime}$ y menor es la $\sigma_{I}$ que como máximo puede aceptar el material. El hormigón trabaja como sometido a un esfuerzo de flexión pura $(\mathrm{T}=0)$, pero con una resistencia útil de compresión $\sigma_{M}$ tanto menor que $\sigma_{b}$ cuanto mayor es el esfuerzo cortante $\mathrm{T}$.

Sea $u$ el coeficiente de corrección

$$
\sigma_{\mathrm{M}}=u \sigma_{b}
$$

que aplicado a la resistencia $\sigma_{b}$. permite deducir la resistencia útil para la flexión $\sigma_{M}$. El valor de $u$, se deduce introduciendo en [4] los valores de $\sigma_{I}$ y $\sigma_{I I}$ que se deducen en función de $\sigma_{\mathrm{M}}$ y de $\tau$. 


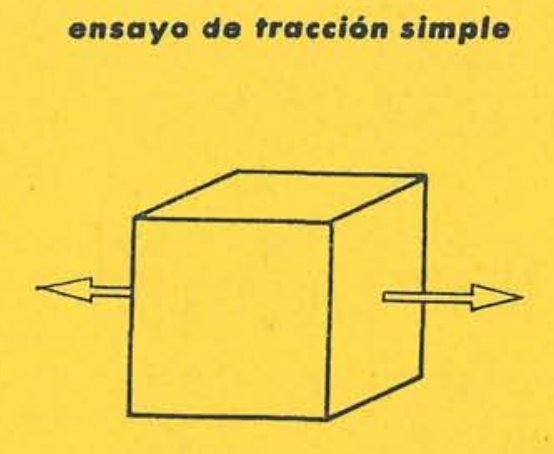

Resistencia en tracción del hormigón $\sigma_{b}$ ensayo de compresión simple

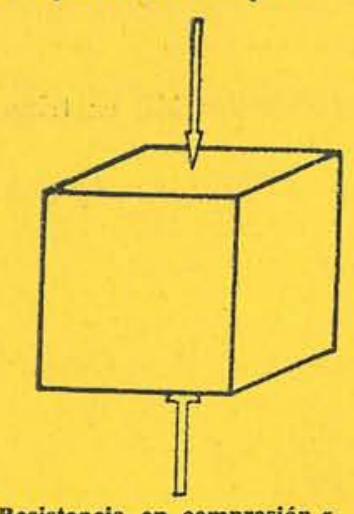

solicitación combinada

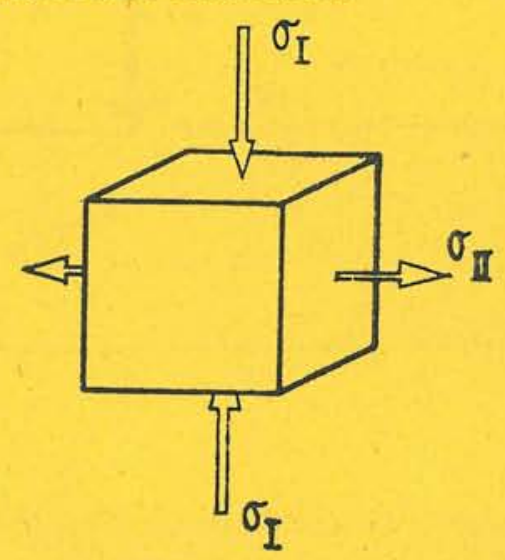

feoría elásica

Hipótesis b)

El hormigón se rompe:

1. Cuando la tensión $\sigma_{1}$ alcanza la resistencia $\sigma_{b}$

2. Cuando la tensión $\sigma_{11}$ sdquiera el valor $\sigma_{b}=0,09 \sigma_{b}$

\section{nueva feoria}

Hipótesis b)

El hormigón se rompe bajo la acción conjunta de of $y \sigma_{\|}$ cuando:

$$
\sigma_{11}=0,09 \sqrt{\sigma_{b}^{2}-\sigma_{1}^{2}}
$$

La expresión analítica que resulta, muy complicada, puede representarse, con muy buena aproximación, por la función:

$$
u=1-10,8\left(\frac{\mathrm{T}}{\mathrm{N}_{\mathrm{b}}}\right)^{2} .
$$

Repitiendo un cálculo análogo con las tensiones principales que resultan de una distribución triangular, y hallando los máximos valores de dichas tensiones, se deduce

$$
u=0,7-0.533 \frac{\mathrm{T}}{\mathrm{N}_{b}}
$$

como expresión suficientemente aproximada, o bien

$$
u=\frac{2}{3}-\frac{1}{2} \frac{\mathrm{T}}{\mathrm{N}_{\mathrm{b}}}
$$

poco diferente de la anterior y que conduce a posteriores simplificaciones.

Entrando con estos valores de $u$ en las fórmulas generales de la flexión pura ( $\mathrm{T}=0$ )

$$
\mathrm{M}=\mathrm{A} \sigma_{e} h_{c}+\mathrm{A}^{\prime} \sigma_{e}^{\prime} h\left(1-\frac{\mathrm{A}^{\prime} \sigma^{\prime}{ }_{e}}{2 \sigma_{\mathrm{M}} b h}\right)
$$


cuando la distribución de tensiones es rectangular y

cuando la distribución es triangular, se deduce:

$$
\mathrm{M}=\mathrm{A} \sigma_{e} h_{c}+\mathrm{A}^{\prime} \sigma_{e}^{\prime} h\left(1-\frac{2 \mathrm{~A}^{\prime} \sigma_{e}^{\prime}}{3 \sigma_{\mathrm{M}} b h}\right)
$$

$$
\mathrm{M}=\mathrm{A} \sigma_{e} h_{c}+h\left(\mathrm{~A}^{\prime} \sigma_{e}^{\prime}-\mathrm{A} \sigma_{e}\right)\left[1-\frac{\mathrm{A}^{\prime} \sigma_{e}^{\prime}-\mathrm{A} \sigma_{e}}{2 \sigma_{b} b h\left[1-10,8\left(\frac{\mathrm{T}}{\mathrm{A}^{\prime} \sigma_{e}^{\prime}-\mathrm{A} \sigma_{e}}\right)^{2}\right]}\right]
$$

y

$$
\mathrm{M}=\mathrm{A} \sigma_{e} h_{c}+h\left(\mathrm{~A}^{\prime} \sigma_{e}{ }_{e}-\mathrm{A} \sigma_{e}\right)\left[1-\frac{\mathrm{A}^{\prime} \sigma_{e}{ }_{e}-\mathrm{A} \sigma_{e}}{\sigma_{b} b h\left(1-\frac{3}{4} \frac{\mathrm{T}}{\mathrm{A}^{\prime} \sigma_{e}^{\prime}-\mathrm{A} \sigma_{e}}\right)}\right]
$$

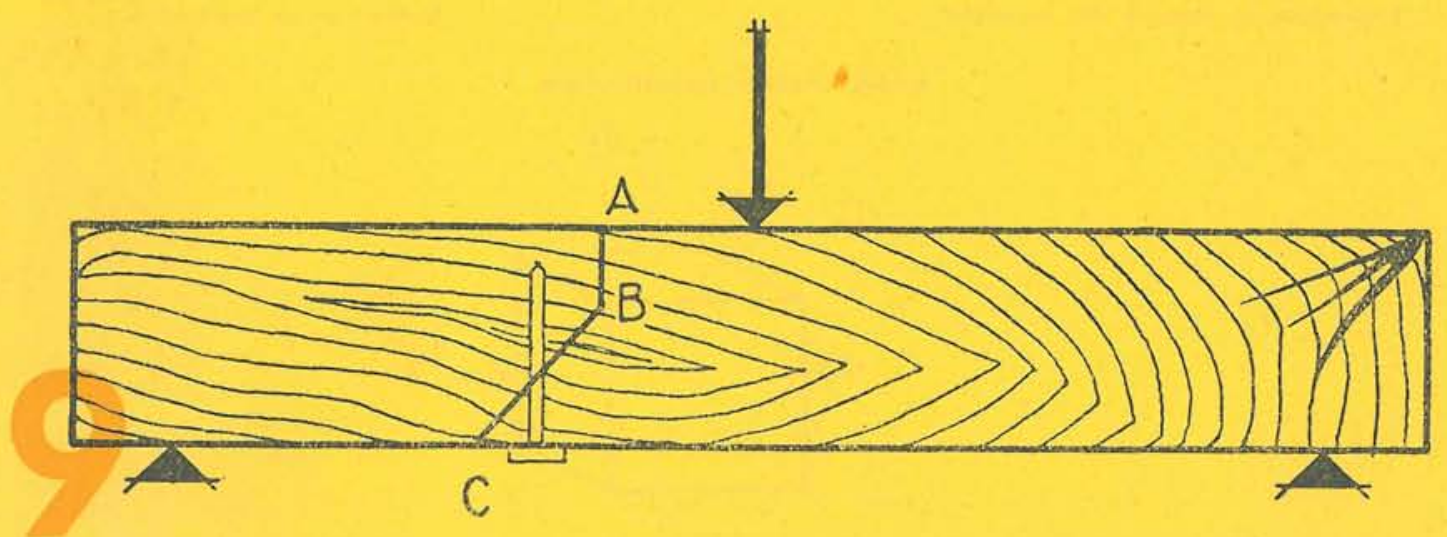

En estas fórmulas, $\mathrm{A}$ representa la sección de las armaduras comprimidas; $\mathrm{A}^{\prime}$, las extendidas; $h_{c}$, la distancia entre baricentros de armaduras en compresión y tracción; $h$, el canto útil; $b$, el ancho de la pieza, y $\sigma_{e}^{\prime}$ y $\sigma_{e}$, el límite elástico de las armad uras. El momento $\mathrm{M}$ es el de rotura.

Ambas expresiones se identifican para:

$$
\frac{\mathrm{T}}{\mathrm{A}^{\prime} \sigma_{e}^{\prime}-\mathrm{A} \sigma_{e}}=0,233
$$

La [11], por consiguiente, se aplicará para valores de $\mathrm{T}:\left(\mathrm{A}^{\prime} \sigma^{\prime}{ }_{e}-\mathrm{A} \sigma_{\iota}\right)$ menores que 0,233 y la segunda para valores mayores.

Operando con estas fórmulas para llegar a expresiones explícitas en la armadura de tracción, se obtiene la expresión simplificada:

siendo:

$$
\mathrm{A}^{\prime} \sigma^{\prime}{ }_{c}=0,97 \frac{\mathrm{M}_{0}}{h}\left(1+\frac{\mathrm{M}_{0}}{u \sigma_{b} b h^{2}}\right)+\mathrm{A} \sigma_{c},
$$

$$
\begin{gathered}
\mathrm{M}_{0}=\mathrm{M}-\mathbf{A} \sigma_{c} h_{c} ₹ 0,375 u \sigma_{b} b h^{2} ; \\
u=1-10,8 \psi^{2} \\
\psi=\frac{\mathrm{Th}}{\mathrm{M}}<0,233, \\
u=0,5-\frac{3}{8} \psi
\end{gathered}
$$$$
\text { si }
$$

en caso contrario.

\section{Piezas de sección rectangular armadas con estribos verticales}

Con el fin de resumir, en pocas líneas, la función desarrollada por los cercos, se indica de modo esquemático el triple efecto que producen: 
1. Los estribos, al reforzar la unión de la cabeza de compresión con el resto de la pieza, coartan la rotura del hormigón mediante un efecto de zunchado análogo al que realizan los cercos en una probeta o soporte de hormigón.

2. Los estribos, al coser la grieta inclinada formada por la acción conjunta del momento flector y del esfuerzo cortante, absorben una importante fracción del esfuerzo cortante.

3. ${ }^{\circ}$ Siendo la grieta inclinada, y considerando la sección oblicua definida por la fisura, los cercos absorben una pequeña fracción del momento flector que actúa. Dos piezas de madera, con la forma que se indica en la figura 9, como representación esquemática de una fisura oblicua abierta en el hormigón, que llega hasta la zona de compresiones $\mathrm{AB}$, y únicamente unidas por un clavo vertical $\mathrm{C}$, permite que el conjunto de las dos piezas acepte un esfuerzo de flexión no despreciable. Si son dos los clavos que cruzan la grieta, el más próximo a $\mathrm{B}$ estará sometido a un esfuerzo de tracción inferior al que soporta el más alejado.

El primer efecto, el de zunchado, equivale, por su acción, a un aumento de la resistencia útil del hormigón; el segundo, a una reducción del esfuerzo cortante que debe absorber el hormigón, con arreglo a lo indicado en el apartado precedente; el tercero, a una reducción del momento flector aplicado.

El primer efecto supone una modificación del coeficiente $u$, que ahora toma la forma:

$$
u=\left(1+5 \frac{\mathrm{A}_{t} \sigma_{e}}{\sigma_{b} b h}\right)\left[1-10,8\left(\frac{\mathrm{T}_{b}}{\mathrm{~A}^{\prime} \sigma_{c}^{\prime}-\mathrm{A}_{c}}\right)^{2}\right]
$$

si

$$
\frac{T_{b}}{A^{\prime} \sigma^{\prime}{ }_{e}-}-\frac{A \sigma_{e}}{A}<0,233
$$

y

$$
u=\left(1+5 \frac{\mathrm{A}_{t} \sigma_{e}}{\sigma_{b} b h}\right)\left[0,5-\frac{3}{8} \frac{\mathrm{T}_{b}}{\mathrm{~A}^{\prime} \sigma^{\prime}{ }_{e}-\mathrm{A} \sigma_{e}}\right]
$$

en caso contrario. En estas expresiones $A_{t}$ es la sección de cercos verticales en una longitud de pieza igual al canto útil $h$.

El segundo efecto, como el tercero, dependen de la inclinación de la fisura, anulándose cuando ésta es vertical $\left(\alpha=90^{\circ}\right)$. Si, siendo

$$
\psi=\frac{\mathrm{T} h}{\mathrm{M}},
$$

se admite que el ángulo que la fisura forma con la horizontal es

y como simplificación

$$
\operatorname{tg} \alpha=\sqrt{1+\frac{1}{\psi}}
$$

$$
\operatorname{tg} \alpha=\frac{1}{\sqrt{\psi}},
$$

se deduce que el esfuerzo cortante $\mathrm{T}_{b}$, que debe resistir la zona de compresiones del hormigón es:

$$
\mathrm{T}_{b}=\mathrm{T}-\mathrm{A}_{t} \sigma^{\prime}{ }_{e} \sqrt{\psi} \text {. }
$$

El tercer efecto, equivale a suponer que los cercos incrementan el momento resistido en la cantidad:

$$
\Delta \mathrm{M}=\frac{\mathrm{A}_{t} \sigma^{\prime}{ }_{e} h \psi}{2} .
$$

Las expresiones [14] a [18] del caso anterior, se transforman ahora en:

siendo:

$$
\mathrm{A}^{\prime} \boldsymbol{\sigma}^{\prime}{ }_{e}=0,97 \frac{\mathrm{M}_{0}}{h}\left(1+\frac{\mathrm{M}_{0}}{u \sigma_{b} b h^{2}}\right)+\mathrm{A} \sigma_{e},
$$

$$
\begin{gathered}
\psi=\frac{\mathrm{T} h}{\mathrm{M}}, \\
\mathrm{M}_{\odot}=\mathrm{M}-\mathrm{A} \sigma_{\iota} h_{c}-\frac{1}{2}-\mathrm{A}_{\iota} \sigma_{e} h \psi<0,375 \sigma_{b} b h^{2} u ;
\end{gathered}
$$


Adoptando un coeficiente de seguridad para las solicitaciones igual a 1,4, como corresponde al caso de daños medios y obras muy vigiladas, las solicitaciones límites son:

$$
\begin{aligned}
& \mathrm{M}=1,4 \times 48=67 \mathrm{~m} . \mathrm{t} . \\
& \mathrm{T}=1,4 \times 11=15,4 \mathrm{t} .
\end{aligned}
$$

Disponiéndose cercos en la proporción

$$
\mathrm{A}_{t} \sigma_{\mathrm{e}}=\frac{1}{2} \mathrm{~T}=7,7 \mathrm{t}
$$

se deduce que la sección de estos cercos es:

$$
\mathrm{A}_{t}=\frac{7,7}{\sigma_{e}}=\frac{7,7}{2,1}=3,8 \mathrm{~cm}^{2} / \mathrm{h} .
$$

Suponiendo que el canto útil es el $90 \%$ del canto total:

$$
\begin{gathered}
h=0,9 \times 0,8=0,72 \mathrm{~m} . \\
\mathrm{A}_{t}=3,8 \mathrm{~cm}^{2} / 0,72 \mathrm{~m}=\frac{3,8}{0,72}=5,3 \mathrm{~cm}^{2} / \mathrm{m} . \mathrm{l} .=10 \varnothing 8 \mathrm{in.} .,
\end{gathered}
$$

es decir, cercos $\varnothing 8$ cada 0,20 metros.

Según las fórmulas [27] a [31]:

$$
\begin{aligned}
& \psi=\frac{15,4 \times 0,72}{67}=0,165 \\
& M_{o}=67-\frac{1}{2}-7,7 \times 0,72 \times 0,165=66,5 \mathrm{~m} . \mathrm{t} . \\
& \psi_{b}=0,165-\frac{7,7 \times 0,72 \sqrt{0,165}}{67}=0,131 \\
& \sigma_{b} b h=2.000 \times 0,25 \times 0,72=360 \mathrm{t} \\
& u=\left(1+\frac{5 \times 7,7}{360}\right)\left(1-10,8 \times \overline{0,131}^{2}\right)=0,9 .
\end{aligned}
$$

La armadura en tracción necesaria es:

$$
\begin{aligned}
& \mathrm{A}^{\prime} \sigma_{\iota}=0,97 \frac{66,5}{0,72}\left(1+\frac{66,5}{0,9 \times 360 \times 0,72}\right)=115 \mathrm{t}, \\
& \mathrm{A}^{\prime}=\frac{115}{2,1}=55 \mathrm{~cm}^{2}=12 \varnothing 25 .
\end{aligned}
$$

En general, la corrección para calcular $\mathrm{M}_{o}$ es despreciable y puede hacerse $\mathrm{M}_{o}=\mathrm{M}$. En realidad, la diferencia con el cálculo corriente se basa en la deducción de $\psi$ y de $u$. El resto de las operaciones son las necesarias para efectuar el dimensionamiento normal.

NOTAS:

Aun cuando en teoría la sección A de armaduras comprimidas debe multiplicarse por el límite elástico $\sigma_{e}$ en compresión del acero, en la práctica se multiplica por el límite elástico en tracción $\sigma_{e}$, dadas las dificultades del ensayo necesario para determinar dicha tensión límite.

El cálculo práctico de las secciones se simplifica notablemente utilizando el ábaco que figura en la publicación monográfica sobre el tema, y que aquí no se incluye por ser un artículo informativo, así como también se omite la concordancia que se obtiene aplicando este método respecto a los resultados experimentales recopilados para comprobar la precisión de esta teorfa. 